\title{
Die Zukunft ärztlicher Führung - Engaging Leadership?
}

\section{Christof Schmitz ${ }^{a}$, Matthias Egger ${ }^{b}$, Peter Berchtold ${ }^{c}$}

a PD Dr. rer. soc. oec., Co-Geschäftsführer college M; b Prof. Dr. med., Institut für Sozial- und Präventivmedizin, Universität Bern;

${ }^{c}$ PD Dr. med., Co-Geschäftsführer college M

Ärztliche Führung hat eine lange Geschichte. Ein Blick über die letzten Jahrzehnte zeigt, dass verschiedene Phasen zu unterscheiden sind - die nächste steht an. Die Frage dabei ist eine doppelte: Erstens, wie kann erfolgreich medizinisches Wissen und Erfahrung in die Gesamtleitung von Spitälern eingebunden werden und zweitens, wie kann eine Führungsform aussehen, die Qualitäts- wie Finanzanforderungen, Patienten- wie Mitarbeiterbedürfnissen gleichermassen entspricht. In der Führungsforschung geht es an dieser Stelle um die Gleichzeitigkeit von Produktivitiät und Well-being. Eine Lösung könnte Engaging Leadership heissen: in Gestalten und Führen eingebunden zu sein, statt als einsamer König von divergierenden Interessen und unüberschaubaren Komplexitäten zerrissen zu werden - und jene Aspekte zu fokussieren, die das Engagement aller erhöhen lassen.

Leadership - nicht Management - war immer Teil der ärztlichen Profession und konnte als integrales Element des professionellen Selbstverständnisses gelten. Im Gestalten von Behandlung, Aus- und Weiterbildung oder Forschung waren seit jeher Ärzte "im Lead» und haben dies als ihrer professionellen Aufgabe inhärent verstanden. Dieses «im Lead sein» scheint stark an Attraktivität eingebüsst zu haben. War Chefarzt werden früher ein häufiges Karriereziel, setzen junge Ärzte und vor allem Ärztinnen heute ganz andere Prioritäten [1]*. Gleichzeitig finden international Diskussionen statt, wie Management und Medizin neu zueinander zu ordnen sind [2]. Hat Gestalten in der Medizin Attraktivität eingebüsst - wo doch eine Mehrheit der Entscheide ärztlich (mit-)verantwortet ist? Und: Wie attraktiv und relevant erscheint ärztliches Gestalten den nicht-medizinischen Kräften?

\section{Eine kleine Geschichte des ärztlichen Führens}

Die ärztliche Gestaltungsrolle hat sich über die Jahrzehnte stark verändert. In den frühen Jahren der modernen Medizin bedeutete ärztliche Leadership Dominanz. Die ärztliche Führung entschied, unterstützt durch einen Verwalter und eine Oberpflegende, praktisch im Allein- gang, allenfalls in Absprache mit Kollegen. Als eigentlicher Vorgesetzter des Chefarztes fungierte die Fachgesellschaft. In den 80er und 90er Jahren gerieten Behandlung und Versorgung komplizierter und Spitalorganisationen komplexer. Management hielt Einzug in die Spitäler und viele Führungspositionen, insbesondere auf Ebene Spitalleitung, wurden mit Betriebswirten besetzt. Es war die Zeit des aufkommenden Glaubens an eine betriebswirtschaftlich geführte Krankenversorgung und ein auf Effizienz getrimmtes Spitalmanagement. Die Führungsrolle der Ärzte dieser Zeit wird in der Literatur mit Repräsentation umschrieben. Chefärztinnen und Chefärzte leiteten ihre Kliniken, auf Stufe Gesamtspital hingegen repräsentierten sie (nur mehr) den ärztlichen Bereich in der Funktion als Mitglieder einer Spitalleitung oder als Medizinische Direktoren.

Vorangetrieben durch die medizinischen Entwicklungen und Innovationen vor allem in den Spezialfächern, sahen sich leitende Mediziner zunehmend nur noch für ihre eigene Disziplin und für den eigenen Bereich (Klinik, Abteilung) verantwortlich. Einher ging damit der Bedeutungszuwachs des Spezialisten-Blicks auf Krankheiten und in der Folge davon eine weitere Fragmentierung von Prozessen und Ergebnissen. Patientenwege folgten jedoch nur begrenzt den Differenzierungen der Spezialitäten und zogen sich mehr und mehr über mehrere disziplinäre Bereiche hin. Nicht zuletzt als Folge davon war eine zunehmende Entkopplung zwischen der ärztlichen Behandlung und der Verantwortung gegenüber dem Gesamten, beispielsweise dem Gesamtergebnis von Behandlung und Betreuung zu beobachten. Accountability hiess im angelsächsischen Raum die Antwort auf diese Entwicklung. Mit Leistungs-Messungen (Performance) sollte die Qualität einzelner medizinischer Massnahmen wie auch ganzer Prozesse und Organisationen gemessen und gesteuert werden. Verschiedene Performance-Messsysteme wurden entwickelt und sind heute in vielen Ländern und an verschiedensten Stellen der Gesundheitsversorgung in Anwendung: Exemplarisch seien die Entwicklung und Anwendung nationaler Qualitätsindikatoren in Norwegen, die bekannten Qualitätsregister in Schwe- 
den, oder auch das Pay-for-Performance-Modell für Hausärzte in England genannt, das einen Fünftel des hausärztlichen Einkommens aufgrund von klinischen, organisatorischen und weiteren Kriterien steuert.

Nur etwas gelang mit den Systemen zur PerformanceMessung höchst ungenügend, nämlich diejenigen, die tagtäglich Patienten betreuten und behandelten in ausreichend motivierender Art einzubinden. Natürlich waren Ärzte und andere Gesundheitsberufe in die Entwicklung und Messung der Performance-Kriterien involviert. Gleichzeitig aber wurden diese PerformanceSysteme in erster Linie von Managern entwickelt und blieben daher insbesondere in Spitälern immer viel stärker Management- (statt ärztlich) orientiert [3]. Resultat war, dass das eigentliche Versprechen der Performance-Systeme, nämlich die Versorgung und ihre Performance zu optimieren und zu steuern, sich nur ansatzweise erfüllte und teilweise auch unerwartete und dysfunktionale Nebenwirkungen hervorbrachten, wie exemplarisch für die Sternen-Ratings von Akutspitälern gezeigt werden konnte [4].

\section{Engaging Leadership}

Eine neue Entwicklung resultiert aus der Beobachtung, dass jene Spitäler die beste Performance zeigen, die sich durch die Integration von medizinischem Fachwissen auf Leitungsebene auszeichnen. «If clinicians can redefine the debate so that it focuses first and foremost on patients and health outcomes, that will provide a strong common purpose for efforts to tackle the challenges of funding structures and access to care.» [5]. Im Kern geht es darum, dass sich Leitung und Management wieder verstärkt auf das Kerngeschäft ausrichten und nicht - bei aller Relevanz - in erster Linie auf Be-

CAS Leadership in Organisationen des Gesundheitssystems Führungskompetenz im medizinischen Kerngeschäft ist erfolgskritisch geworden. Das ist heute evidenzbasiert. Der Studiengang "CAS Leadership in Organisationen des Gesundheitssystems" vermittelt speziell eine an Organisationen des Gesundheitswesens und ihren Entwicklungen orientierte Leadership. Das Programm verbindet evidenzbasiertes Wissen mit praktischen Anwendungen in einer anregenden Lernkultur.

Der Studiengang wird vom Institut für Sozial- und Präventivmedizin der Universität Bern und vom college M durchgeführt. Er richtet sich an Ärzte und Ärztinnen mit leitenden Funktionen sowie Führungskräfte anderer Berufsgruppen in Spitälern und weiteren Einrichtungen.

Der Studiengang gliedert sich in 6 Module von insgesamt 18 Tagen und eine Projektarbeit. Der Studiengang wird mit einem "Certificate of Advanced Studies (CAS) in Leadership in Health Care Organisations» der Universität Bern (15 ECTSPunkte) abgeschlossen. Detaillierte Informationen und Anmeldung: www.cas-leadership.ch oder Tel. 0316313511.
Tabelle 1: Vergleich Engagement und Performance NHS Trusts [7].

\begin{tabular}{|c|c|c|c|c|}
\hline & Trust ID & $\begin{array}{l}\text { Medical } \\
\text { Engagement } \\
\text { Index }\end{array}$ & $\begin{array}{l}\text { Overall } \\
\text { quality } \\
\text { score }\end{array}$ & $\begin{array}{l}\text { Financial } \\
\text { management } \\
\text { score }\end{array}$ \\
\hline \multirow{4}{*}{$\begin{array}{l}\text { Top } 4 \\
\text { trusts }\end{array}$} & 21 & 65.8 & Good & Excellent \\
\hline & 12 & 65.2 & Good & Good \\
\hline & 15 & 63.4 & Excellent & Good \\
\hline & 5 & 62.0 & Excellent & Excellent \\
\hline \multirow{4}{*}{$\begin{array}{l}\text { Bottom } 4 \\
\text { trusts }\end{array}$} & 26 & 53.1 & Fair & Fair \\
\hline & 8 & 52.7 & Good & Good \\
\hline & 18 & 52.1 & Fair & Fair \\
\hline & 20 & 47.0 & Poor & Poor \\
\hline
\end{tabular}

triebswirtschaft und Ökonomie. Wenn solches Medical Engagement oder Engaging Leadership in Spitälern gelingt, dafür spricht eine wachsende Evidenz, sind die qualitativen wie finanziellen Ergebnisse besser. Profit will follow social profit, lautet die Devise [6].

Beispielhaft sei das an einer englischen Arbeit gezeigt, die den Zusammenhang zwischen Medical Engagement und qualitativer bzw. finanzieller Performance untersucht hat und zeigen konnte, dass mit höherem Medical Enagegement Index bessere Qualitäts- wie Finanz-Scores erzielt werden (Tab. 1).

Die Patientenbehandlung und Versorgung der Zukunft wird sich durch eine noch viel höhere Komplexität auszeichnen. Einer Komplexität, der nur jene Spitäler gewachsen sein werden, denen es gelingt Engagement aufzubauen. Engaging leadership in Spitälern zeichnet sich dadurch aus, dass Leitungsgremien die Besonderheiten der Patientenbehandlung und des Versorgungssystem, die Möglichkeiten und Risiken der Medizin, die Unterschiede zwischen Patienten(-Gruppen) und in den universitären Häusern das Nebeneinander von Klinik, Lehre und Forschung in ihre Entscheidfindung «engagierend» einbeziehen. Strukturelle Einbindung reicht allerdings nicht. Die konkrete Art der Führung ist gleichermassen von Bedeutung. Engaging Leadership umfasst ausdrücklich auch das Wie der Führung, nicht nur das Was und Wer [8].

Engaging Leadership verlangt, dass sich Medizin und Mediziner «engagiert» auf allen Leitungsebenen in die Entscheidfindung einbringen und entsprechende gemeinsame Verantwortung übernehmen. Das stellt für viele Mediziner eine grosse Herausforderung dar: Als Ärzte wurden sie traditionell für individuelles Handeln (gegenüber dem Patienten) ausgebildet. Handeln als Team und sich als Organisation Verstehen ist in der ärztlichen Aus- und Weiterbildung noch immer kaum Thema. In Führung und Leadership steht das aber ganz besonders im Fokus. Engaging Leadership - von Ärzten erwartet! 


\section{Literatur}

1 van den Bussche $\mathrm{H}$, Wonneberger C, Birck S, Schultz JH, Robra BP, Schmidt A, et al. Die berufliche und private Situation von Ärztinnen und Ärzten zu Beginn der fachärztlichen Weiterbildung. Gesundheitswesen. 2014;76:e1-e6.

2 Kuhlmann E, Burau V, Correla T, Lewandowski R, Lionis C, Noordegraaf M, Repullo J. "A manager in the minds of doctors:» a comparison of new modes of control in European hospitals. BMC Health Services Research. 2013,13:246.

3 Smith PC. Performance Measurement in Health Care:

History, Challenges and Prospects. Public Money and Management. 2005;25:213-20.
4 Mannion R, Davies H, Marshall M. Impact of star performance ratings in English acute hospital trusts. J Health Serv Res Policy. 2005;10:18-24.

5 Darzi A. A time for revolutions: the role of clinicians in health care reform. The New England Journal of Medicine. 209;361(6):e8.

6 Mayo. Lessons ... BITTE NOCH ERGÄNZEN

7 Spurgeon P, Clark J, Ham C. Medical Leadership. Radcliff; 2011.

8 Engaging Leadership-Creating Organisations that Maximise the Potential of their People - written by Professor Beverly AlimoMetcalfe and John Alban-Metcalfe and published by the Chartered Institute of Personnel and Development. 